\title{
A FULLY THREE-DIMENSIONAL BAYESIAN IMAGE RECONSTRUCTION FOR SMALL ANIMAL PINHOLE SPECT
}

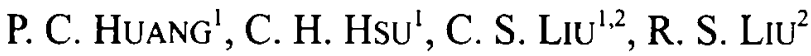 \\ 'Department of Nuclear Science, National Tsing Hua University, Hsinchu \\ ${ }^{2}$ National PET/Cyclotron Center, Taipei Veterans General Hospital \\ Taiwan
}

\begin{abstract}
This paper presents a fully 3D Bayesian image reconstruction and its localization properties of a pinhole SPECT for small animal imaging. Due to the unique geometric characteristics, pinhole SPECT for small animals can use existing clinical equipment to produce high resolution image results. However, low sensitivity is the major disadvantage of pinhole SPECT and leads to instability of iterative image reconstruction based on popular maximum likelihood expectation and maximization (MLEM). In this work, we describe a fully 3D system model of a pinhole SPECT combined with an image model based on medium root prior (MRP). Under the paradigm of Bayesian statistics, a modified EM algorithm with one-step late (OSL) is applied to reconstruct $3 D$ SPECT images through maximum a posteriori (MAP) estimation. The experimental results of phantom studies indicate that the proposed 3D Bayesian image reconstruction can produce better edgepreserving image results and effectively suppress noises.
\end{abstract}

Biomed Eng Appl Basis Comm, 2004 (August); 16: 180-184.

Keywords: Pinhole Animal SPECT, Maximum Likelihood Expectation Maximization, Median Root Prior, Bayesian Image Reconstruction.

\section{INTRODUCTION}

Developments in clinical research such as gene therapy have resulted in an increased interest in the in vivo imaging of small animals. Dedicated systems for imaging small animals have been designed for some imaging modalities such as MRI, CT, and PET. Pinhole SPECT is also an essential modality because it offers the possibility of imaging gene expression or radio-

Received: March 1, 2004; Accepted: July 25, 2004

Correspondence: Ching-Han Hsu, Ph.D.

Department of Nuclear Science, National Tsing Hua

University, 101, Section 2, Kuang Fu Road,

Hsinchu 300, Taiwan.

E-mail:cghsu@mx.nthu.edu.tw labeled ligands with specific receptor binding [1].

One advantage of pinhole SPECT is that a general-purpose gamma camera can be used for ultrahigh-resolution experiments for in vivo imaging with small animals [1-4]. Because of the converging beam geometry of pinhole collimator (Fig. 1), pinhole SPECT can provide high resolution functional images. This modality has recently received growing interest for the purpose of imaging small laboratory animals. Recent advances have identified the technology that can be used to provide images with spatial resolution of the order of $1 \sim 3 \mathrm{~mm}$ [5].

Since SPECT is primarily a clinical tool designed to image humans, pinhole SPECT suffers the disadvantages of low sensitivity and requires special image methods to reconstruct image volumes of small animal. Conventional algebraic methods using filtered back-projection (FBP) can not count for this photon- 
limited nature of pinhole SPECT data, and may result into unsatisfactory image results with streaking artifacts. [6]

Alternatively, statistical SPECT image estimation based on Poisson model can well portrait the discrete nature of single photons. The corresponding iterative reconstructions using maximum likelihood expectation and maximization (MLEM) have been demonstrated to generate superior image results compared to FBP. Even so, for the cases of low sensitivity (or very low count rate), the image results inevitably become deteriorated as the number of iteration increases, due to the noise contamination. The ill-posed naturc of ML estimation is another major cause of algorithm divergence [7].

The noise accumulation in pinhole SPET images could be reduced by using Bayesian reconstruction methods. Smoothing image prior can be used to regularize the solution of the reconstruction problem [7]. Furthermore, to prevent edges from being blurred, we consider the use of the median root prior (MRP) for fully 3D Bayesian image reconstruction [8-9]. The median filter in MRP is dependent only on one parameter that defines a neighborhood system. MRP also prefer locally monotonic images by removing very large or small isolated voxel values as noise leaving edges unaltered.

The aim of this paper is to develop a fully $3 D$ Bayesian image reconstruction of small animal pinhole SPECT. In section II, we describe the statistical model for the data and image, and present details of reconstruction methods. Results and discussions are presented in Section III for two phantom studies. Conclusion is contained in Section IV.

\section{METHOD}

\subsection{System Model of 3D Pinhole SPECT}

Due to the converging beam geometry (Fig. 1), pinhole camera can provide high resolution images of targets close to the focal point. The pinhole magnification allows a spatial resolution of reconstructed images below the intrinsic resolution of the gamma camera. To avoid possible artifacts and loss of resolution, the reconstruction of data collected with a pinhole camera requires an accurate description of the camera geometry.

In this paper, we construct a 3D system matrix $\mathbf{P}$ to describe the relation the unknown photon-emission source (image) $\lambda$, and the average collected event $\overline{\mathbf{y}}$ as

$$
\overline{\mathbf{y}}=\mathbf{P} \boldsymbol{\lambda}
$$

That is, the average count for each three dimensional line of response $\bar{y}_{i}$ of pinhole SPECT is related to $\lambda$ as:

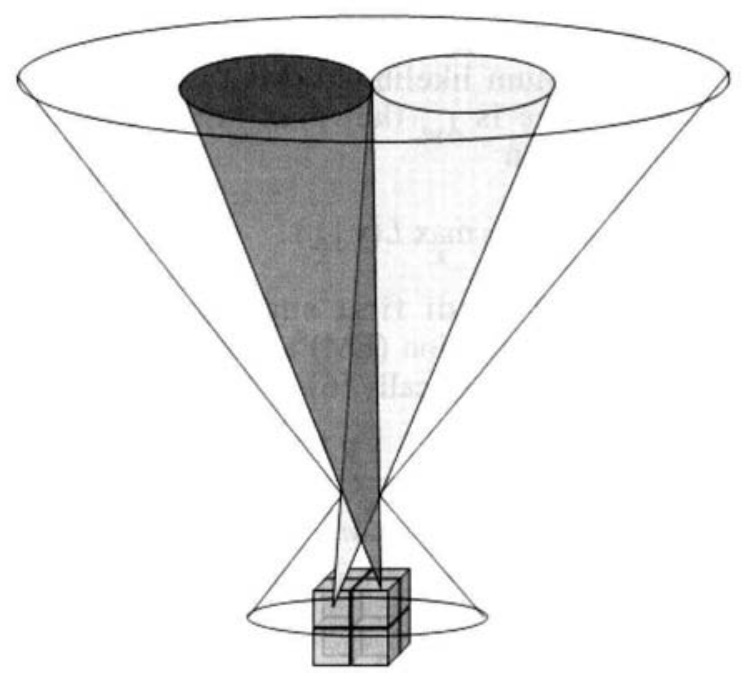

Fig 1. The schematic diagram of a threedimensional pinhole SPECT system for small animal imaging.

$$
\bar{y}_{i}=\sum \mathrm{P}_{i j} \lambda_{j}
$$

The system matrix $\mathbf{P}$ is basically designed according to the physical geometry of pinhole SPECT. The elements of the matrix indicate the probability of one photon emitted form the source, trans-passing the pinhole, and detected by a detector. We assume each incident photon can be probably detected four neighboring detectors adjacent to the location where photon reaches the detector plate in a fully $3 \mathrm{D}$ manner [9]. Fig. 2 shows the schematic diagram of photon detection.

\subsection{Maximum Likelihood Estimation}

In order to capture the photon limited nature of the pinhole SPECT data, the detected photons are modeled as a set $\mathbf{y}$ of independent Poisson random variables with mean $\overline{\mathbf{y}}$ [6]. Therefore, the conditional probability distribution of a pinhole SPECT measurement is

$$
P(\mathbf{y} \mid \lambda)=\prod_{i} \exp \left(-\bar{y}_{i}\right)\left(\bar{y}_{i}\right)^{y_{i}} /\left(y_{i} !\right)
$$

The corresponding logarithmic likelihood function is

$$
L(\lambda)=\ln P(\mathbf{y} \mid \lambda)=\sum_{i} y_{i} \ln \bar{y}_{i}-\bar{y}_{i}
$$

i.e.

$$
L(\lambda)=\sum_{i} v_{i} \ln \left(\sum_{j} \mathrm{P}_{i j} \lambda_{j}\right)-\left(\sum_{j} \mathrm{P}_{i j} \lambda_{j}\right) .
$$


Notice that the constant term equal to $\sum_{i} \ln y_{i}$ is omitted since it is independent of $\lambda$.

The maximum likelihood (ML) estimate of the unknown image is $\hat{\lambda}_{M L}$ that maximizes the above likelihood function

$$
\hat{\lambda}_{M L}=\max _{i} L(\mathbf{y} \mid \lambda) \text {. }
$$

Shepp and Vardi first suggested using the expectation maximization (EM) algorithm to calculate the ML estimate numerically [6]

$$
\lambda_{j}^{k+1}=\lambda_{j}^{k} \sum_{i} \frac{y_{i} \mathrm{P}_{i j}}{\sum_{l} \mathrm{P}_{i l} \lambda_{l}^{k}},
$$

where $k$ is the iteration number.

\subsection{Bayesian Estimation}

Since MLEM tends to diverge for low-sensitivity pinhole SPECT, a more plausible approach is incorporate an image prior model that can (i) regularize the ML estimation and (ii) suppress noises. If a suitable prior model can be formulated to describe these expected characteristics of the unknown image, a Bayesian image reconstruction can then formulated. Here we assume the image prior model is characterized by a Markov random field (MRF) with the following Gibbs distribution [7]

$$
\operatorname{Pr}(\lambda)=\frac{1}{Z} \exp (-\beta U(\lambda)),
$$

where $\beta$ is the hyperparameter that influences the degree of smoothness of the estimated images, $U(\lambda)$ is the Gibbs energy function, and $Z$ is a normalization constant or partition function. In the results presented below, we vary $\beta$ to produce images with a range of background variance levels.

In this work, we consider the median root prior (MRP). The energy function $\operatorname{Med}\left(\lambda_{j}\right)$ of MRP is the medium of image voxel intensities over a nearest neighborhood centered at voxel $j$. The neighborhood system, consisting of $3 \times 3 \times 3$ voxels, is shown Fig. 3 . Median filter favors local monotonic images, removes isolated hot or cold spots (known as pepper and salt noises), and leaves edges unaltered [8-9].

By Bayesian theorem, the posterior density of the unknown image $\lambda$ is

$$
P(\lambda \mid \mathbf{y})=\frac{P(\mathbf{y} \mid \lambda) P(\lambda)}{P(\mathbf{y})} .
$$

The image reconstruction becomes the Bayesian estimation or maximum a posteriori (MAP) estimation searching for the optimal $\hat{\lambda}_{M A P}$ that maximizes the logarithmic posterior function

$$
\hat{\lambda}_{M A P}=\max _{\lambda} \log P(\lambda \mid \mathbf{y})
$$

The following modified EM algorithm with one-step-late (OSL) is applied to compute the $\hat{\lambda}_{\text {MAP }}$ iteratively [8-10]. The update equation of MAP. MRP is given as

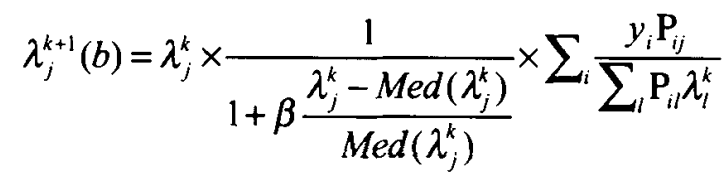

\section{RESULTS AND DISCUSSIONS}

Consider a pinhole SPECT system with a $50 \times 50$ detector array and sizes of each detector equal to $2.2 \mathrm{~mm}$ $\times 2.2 \mathrm{~mm}$. Distance between object and pinhole is $16 \mathrm{~mm}$, while distance between detector plan and pinhole is 90 $\mathrm{mm}$. There are total 128 angular projections (2.8 degrees per projection). The $3 \mathrm{D}$ image volume has $32 \times 32 \times$ 32 voxels with voxel sizes $0.3 \times 0.3 \times 0.3 \mathrm{~mm}^{3}$.

We first consider a uniform spherical phantom with radius equal to 12 voxels as shown in Fig. 4 There is 50:1 intensity ratio between the spherical object and its background. The uniform phantom is first forward-projected and the corresponding sinogram is then sampled using Poisson random number generator. Based on the sampled sinogram, both MLEM and MAP-MRP are used to reconstruct the original spherical image. Twenty iterations are applied to both algorithms. And different values of

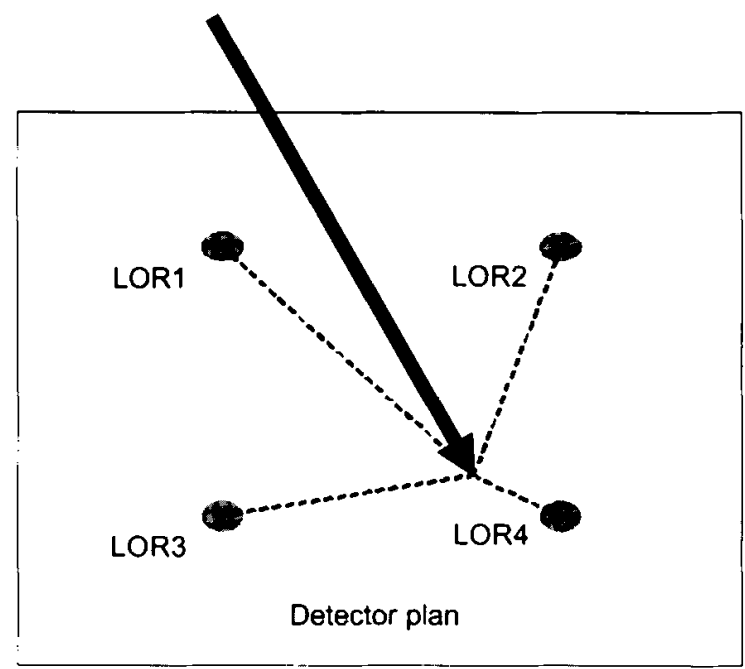

Fig 2. The relationship between one incident photon with four neighboring detectors. Each detector connecting with pinhole forms a single line of response (LOR). 


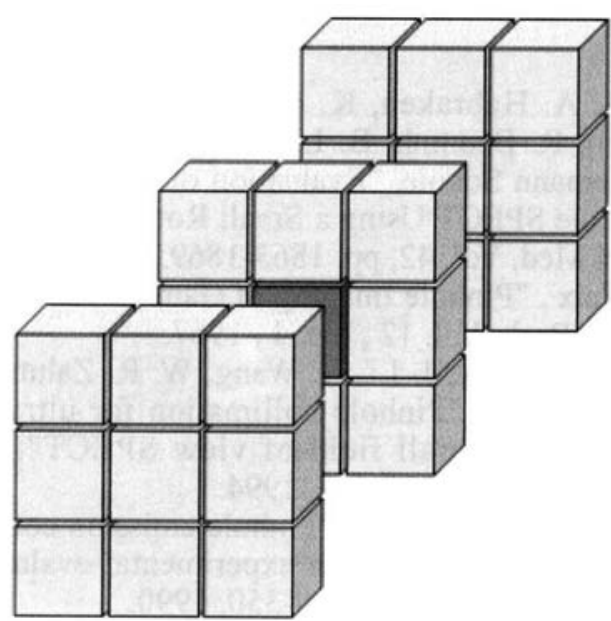

Fig 3. A voxel (at the center of the middle slice) and its 26 nearest neighbors for a $3 \mathrm{D}$ image prior system.
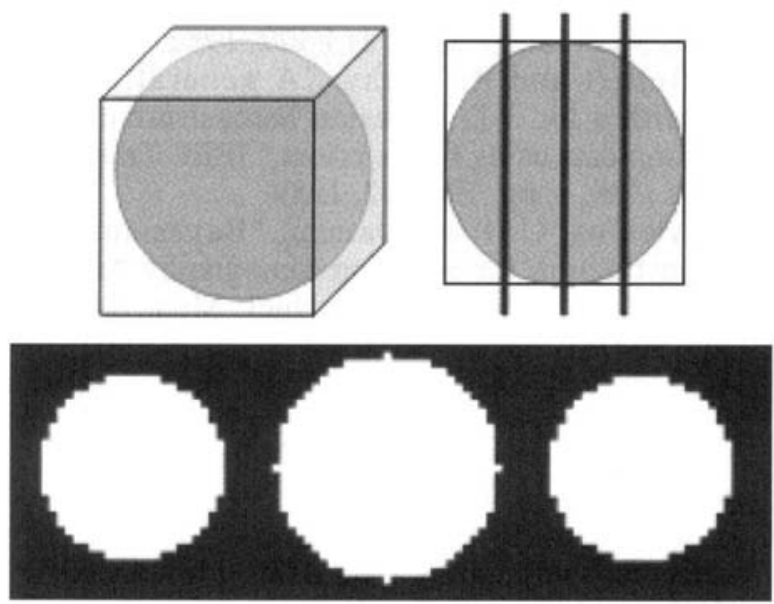

Fig 4. Top row: a uniform spherical phantom and its side view. Bottom row: the corresponding slice views (from left to right) at the $8^{\text {th }}$, the $16^{\text {th }}$, and $24^{\text {th }}$, respectively.

hyperparameter $\beta$ are also applied to MAP-MRP.

Fig. 5 shows image results reconstructed by MLEM and MAP-MRP with $\beta$ equal to $0.1,0.3$, and 0.5 , respectively. The MLEM result has a large degree of variation inside the uniform spherical phantom, while MAP-MRP results visually demonstrate better noise suppression. Shown in Fig. 6 is the plot of profiles of the original phantom, MLEM, and MAPMRP with $\beta=0.3$. The variation inside the uniform phantom is greatly suppressed, while the edges

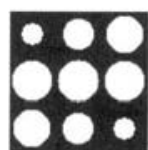

(a)

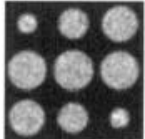

(b)

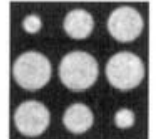

(c)

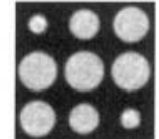

(d)

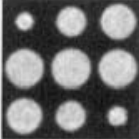

(e)
Fig 5. The reconstructed image results of the uniform phantom: (a) the original phantom, (b) MLEM, (c) MAP-MRP with $\beta=0.1$, (d) MAPMRP with $\beta=0.3$, (e) MAP-MRP with $\beta=0.5$.

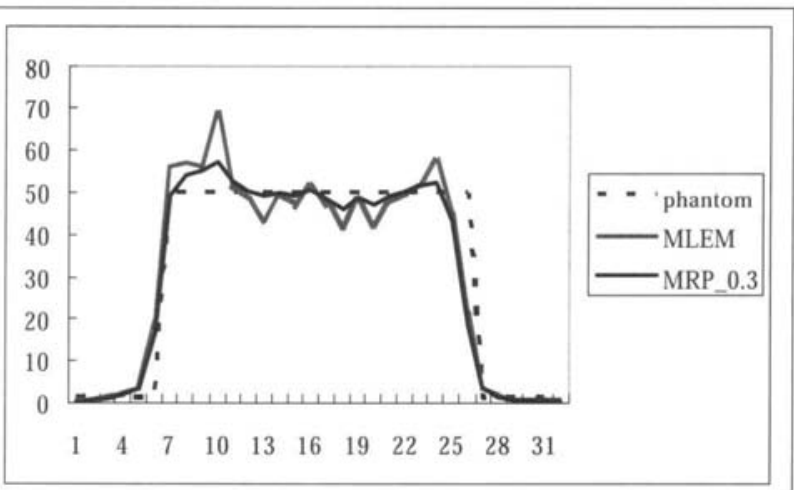

Fig 6. The profiles of central slice for uniform phantom reconstructed using MLEM and MAPMRP with $\beta=0.3$.

between objects and background are preserved. This quality improvement is mainly due to the nice edgepreserving property of median root prior.

Fig. 7 shows another phantom similar to the previous one. In addition, there are five more lesions with radius 5 voxels each. The intensities of these lesions are $25,100,125,150$, and 200 , respectively. These lesions are meant to simulate the situations that both cold and hot lesions exist. By repeating the same reconstruction procedures as the above case, the image results are shown in Fig. 8. Once more, the visual comparison of image results has indicated the suggested MAP-MRP can produce smoother image results while maintaining various heights of edges in the phantom.

\section{CONCLUSION}

In this work, we described a fully 3D system model of a pinhole animal SPECT and a medium root prior as image model. Under the paradigm of Bayesian statistics, the reconstruction of 3D images are achieved by the MAP-MRP estimation which maximizes the posterior density function using a modified EM 


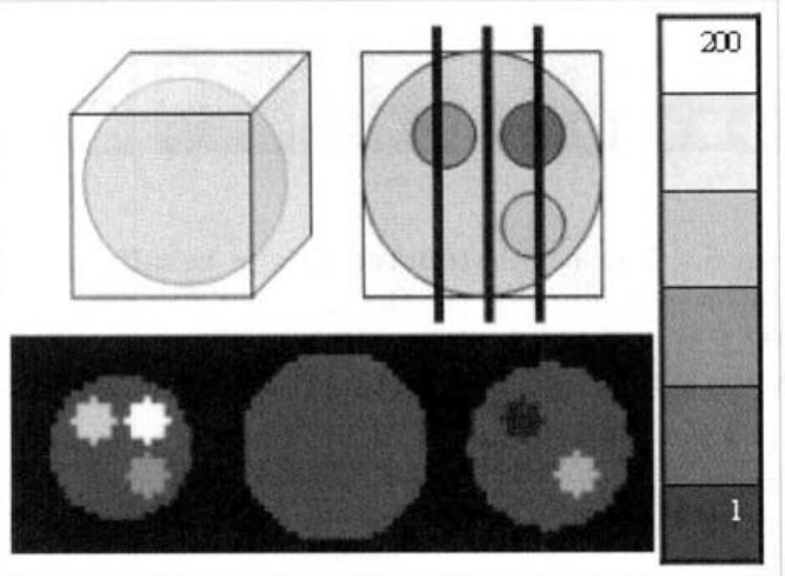

Fig 7. Top row: a non-uniform spherical phantom and its side view. Bottom row: the corresponding slice views (from left to right) at the $8^{\text {th }}$, the $16^{\text {th }}$, and $24^{\text {th }}$, respectively.

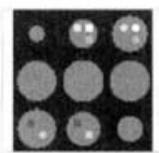

(a)

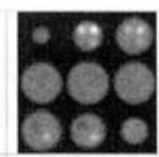

(b)

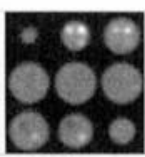

(c)

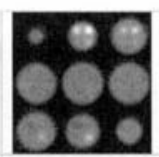

(d)

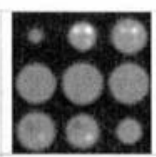

(e)
Fig 8. The reconstructed image results of the nonuniform phantom: (a) the original phantom, (b) MLEM, (c) MAP-MRP with $\beta=0.1$, (d) MAPMRP with $\beta=0.3$, (e) MAP-MRP with $\beta=0.5$.

algorithm with one-step late. Experimental results have indicated that the proposed framework of Bayesian image reconstruction can preserve image edges while effectively suppressing image noises. The proposed framework can then provide more stable image results for high-resolution small animal imaging using 3D pinhole SPECT. For the future routine practice, other accelerated iterative algorithms, like ordered subsets expectation maximization (OSEM) [11], should be implemented to further reduce reconstruction time.

\section{ACKNOWLEDGEMENT}

This research work was supported in part by the National Science Council, Taiwan, (NSC 92-2218-E007-025, and NSC 93-NU-7-007-006), and in part by Tsou's Foundation of Veteran General Hospital (VGHUST93-G6-06).

\section{REFERENCE}

1. J. B. A. Habraken, K. de Bruin, M. Shehata, J. Booij, R. Bennink, B. L. F. van Eck Smit, and E. Busemann Sokole, "Evaluation of High-Resolution Pinhole SPECT Using a Small Rotating Animal," J Nucl Med, Vol. 42, pp. 1863-1869, 2001.

2. D. Paix , "Pinhole Imaging of Gamma Rays", Phys. Med. Biol., Vol. 12 , No. 4, 1967.

3. R. J. Jaszczak, J. Li, H. Wang, W. R. Zalutsky, R. E. Coleman, "Pinhole collimation for ultra-highresolution, small field-of view SPECT", Phys. Med. Biol., 39, 425-437, 1994.

4. J. Palmer, P. Wollmer, "Pinhole emission computed tomography: method an experimental evaluation", Phys. Med. Biol., 35, 339-350, 1990.

5. L. R. MacDonald, B. E. Patt, J. S. Iwanczyk, B. M. W. Tsui, Y. Wang, E. C. Frey, D. E. Wesscll, P. D. Acton, and H. F. Kung, "Pinhole SPECT of mice using the LumaGEM gamma camera," IEEE Trans Nucl. Sci., Vol. 48, pp. 830-836, 2001.

6. L. A. Shepp, Y. Vardi, "Maximum likelihood reconstruction for emission tomography", IEEE Trans Med. Imag., Mi-1, 113-122, 1982.

7. T. Hebert and R. Leahy, "A generalized EM algorithm for 3-D Bayesian reconstruction from Poisson data using Gibbs priors," IEEE Trans Med. Imag., Vol. 8, pp. 194-202, 1989.

8. S. Alenius, U. Ruotsalainen, "Bayesian image reconstruction for emission tomography based on median root prior", Eur. J. Nucl. Med., 24, 256$265,1997$.

9. A. Sohlberg, J. T. Kuikka, U. Ruotsalainen, "Pinhole single phantom emission tomography reconstruction based on median root prior", Eur., J., Nucl., Med., 30, 217-221, 2003.

10. P. J. Green, "Bayesian reconstructions from emission tomography data using a modified EM algorithm," IEEE Trans Med. Imag., Vol. 9, pp. 8493, 1990.

11. H. M. Hudson and R. S. Larkin, "Accelerated Image Reconstruction Using Ordered Subsets of Projection Data", IEEE Trans Med. Imag., Vol. 13, No. 4, pp. 601-609, 1994. 